\title{
Alissonotum piceum besucheti Endrödi, 1977, espèce nouvelle pour I'île de la Réunion" (Coleoptera, Dynastidae)
}

Bernard Vercambre, Didier Pastou, Henri-Pierre Aberlenc, Ronald Manikom

\section{Résumé}

Détecté depuis 2000 par des adultes capturés au piège lumineux, une première observation des larves d' Alissonotum piceum besucheti dans un champ de canne à sucre en 2005 , confirme l'implantation de l'espèce à la Réunion. Des données biologiques sont présentées, ainsi qu'une réflexion sur les voies possibles de son introduction et sa capacité de nuire aux cultures de l'île.

\section{Abstract}

Alissonotum piceum besucheti Endrödi, 1977 : a new introduction in Reunion Island (Coleoptera, Dynastidae).

Detected since 2000 by adults collected in light-trap, a first observation of the larvae has been made in a sugar cane field in Réunion island during 2005. Biological data, as considerations on its introduction ways and pest status in relation with the island agricultural production are presented.

\section{Citer ce document / Cite this document :}

Vercambre Bernard, Pastou Didier, Aberlenc Henri-Pierre, Manikom Ronald. Alissonotum piceum besucheti Endrödi, 1977, espèce nouvelle pour l'île de la Réunion" (Coleoptera, Dynastidae). In: Bulletin de la Société entomologique de France, volume 112 (3), septembre 2007. pp. 349-356;

https://www.persee.fr/doc/bsef_0037-928x_2007_num_112_3_16444

\section{Ressources associées :}

Alissonotum piceum besucheti

Fichier pdf généré le 07/10/2019 


\title{
Alissonotum piceum besucheti Endrödi, 1977, espèce nouvelle pour l'île de la Réunion (Coleoptera, Dynastidae)
}

\author{
par Bernard VERCAMBRE*, Didier PASTOU**, Henri-Pierre ABERLENC*** \\ et Ronald MANIKOM****
}

*CIRAD TA B-05/02 avenue Agropolis, UR Systèmes Canniers, F - 34398 Montpellier cedex 5 <bernard.vercambre @cirad.fr> **CIRAD, UMR C53 PVBMT, Pôle Protection des Plantes, 7 chemin de l'IRAT, F - 97410 Saint-Pierre, Réunion ***CIRAD TA A-55/L, Campus de Baillarguet (CSIRO), UMR CBGP, F - 34398 Montpellier cedex 5 Jhenri-pierre.aberlenc@cirad.fr> **** SPV Aéroport Roland-Garros, Frêt, BP 14, F - 97438 Sainte-Marie, Réunion

Résumé. - Détecté depuis 2000 par des adultes capturés au piège lumineux, une première observation des larves d'Alissonotum piceum besucheti dans un champ de canne à sucre en 2005 , confirme l'implantation de l'espèce à la Réunion. Des données biologiques sont présentées, ainsi qu'une réflexion sur les voies possibles de son introduction et sa capacité de nuire aux cultures de l'île.

Summary. - Alissonotum piceum besucheti Endrödi, 1977 : a new introduction in Reunion Island (Coleoptera, Dynastidae). Detected since 2000 by adults collected in light-trap, a first observation of the larvae has been made in a sugar cane field in Réunion island during 2005. Biological data, as considerations on its introduction ways and pest status in relation with the island agricultural production are presented.

Keywords. - Coleoptera, Dynastidae, Alissonotum piceum, Reunion Island, Mascarene Islands, new introduction, extension, biology, sugar cane.

\section{Faunistique et spéciation}

Alissonotum piceum Fabricius, 1775 (Dynastidae, Pentodontini) est un Coléoptère répandu dans la région orientale (Inde, Myanmar, Sri Lanka). Les îles Mascareignes sont incluses dans la sous-région malgache de la région afrotropicale, à la limite entre cette région et la région orientale. A. piceum est connu de l'île Maurice (BOX, 1953 ; WILLIAMS \& MAMET, 1962). On ignore la date de son introduction, mais la morphe des Mascareignes étant différenciée par rapport à la forme-type, il est donc probable que cette colonisation soit relativement ancienne. En effet, le taxon présent à Maurice et récemment découvert à la Réunion, a été élevé au rang de sous-espèce par ENDRÖDI (1977 et 1985) sous le nom d'Alissonotum piceum besucheti (fig. 1) en s'appuyant sur plusieurs caractères, dont le principal est la forme des paramères de l'édéage (fig. 4). Pour différencier les sexes dans un échantillon d'adultes, il faut examiner la face ventrale de l'abdomen : le mâle présente une échancrure au dernier sternite abdominal (fig. 2) alors qu'il est régulièrement arrondi (fig. 3) chez la femelle (DECHAMBRE, 1986). Enfin, le raster de la larve est caractéristique de certains Dynastidae qui ne présentent aucun arrangement régulier des poils (absence de palidia) (fig. 6). La détermination a été faite indépendamment par R.-P. Dechambre au MNHN', Paris, par M. Lacroix et par l'un de nous (HPA).

Le statut du taxon mauricien peut servir à raisonner le temps d'évolution vers la spéciation progressive d'un insecte car on dispose de repères chronologiques précis liés à la mise en culture cannière de l'île, longtemps inhabitée. Les Hollandais s'y installèrent provisoirement en 1598. L'introduction de la Canne date de la période 1639-1650 (RoullLARD, 1990). Mais ce n'est qu'en 1735 que l'on signale les débuts d'une culture semi-industrielle, résultat de multiples importations de Canne sous toutes ses formes. Ainsi, la différenciation morphologique de la sous-espèce besucheti se serait produite au cours des 2,5 à 3 siècles précédents.

\footnotetext{
I Abréviations des différentes institutions citées. - MNHN : Muséum national d'Histoire naturelle, Paris, France. FDGDON : Fédération Départementale des Groupements de Défense contre les Organismes Nuisibles, Saint-Paul, Réunion. SPV: Service de Protection des Végétaux, Saint-Denis, Réunion. CIRAD: Centre de Coopération Internationale en Recherche Agronomique pour le Développement, Saint-Pierre, Réunion. MSIRI : Mauritian Sugar Industry Research Institute, Le Réduit, Maurice. DAF : Direction de l'Agriculture et des Forêts, Saint-Denis, Réunion.
} 


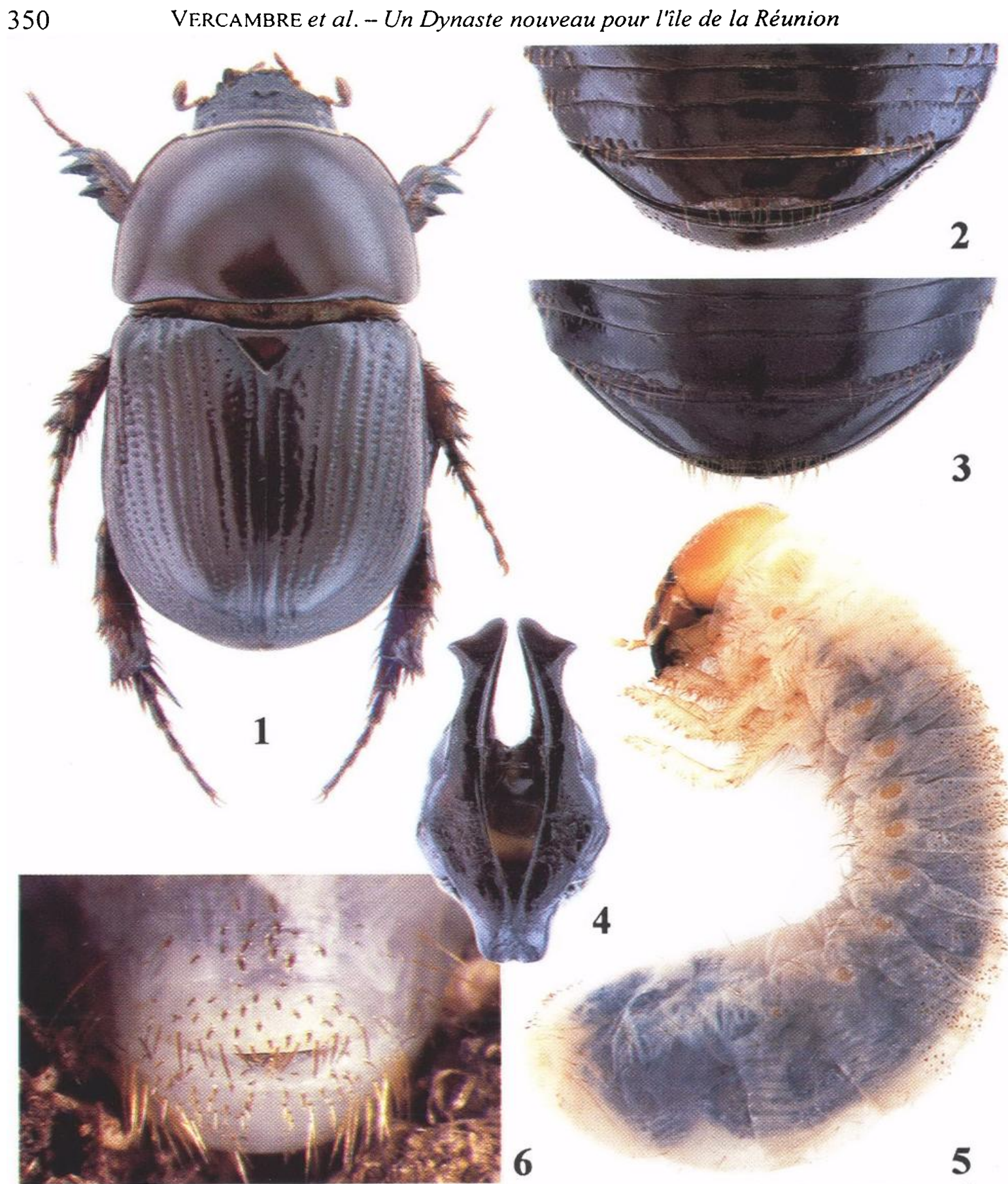

Fig. 1 à 6. - Alissonotum piceum besucheti. - 1, Adulte. -2 et 3 , Pygidium $\widehat{\partial}$ et $\bigcirc .-4$, Edéage, $\widehat{\partial} .-5$, Larve de $3^{\mathrm{e}}$ stade. -6 . Raster.

\section{Premières observations de Alissonotum piceum à la Réunion}

$A$. piceum besucheti a été découvert à la Réunion en trois lieux différents situés en bord de mer: des adultes vus pour la première fois à Sainte-Marie (aéroport Roland-Garros, à Gillot) en décembre 2000 , au piégeage lumineux installé en application du protocole de coopération bilatéral franco-mauricien; une $Q$ adulte vivante, sur un parking à Saint-Leu le 17 février 2002 ( $J$. Rochat leg., R.-P. Dechambre det.); $1 \delta, 2$ 을 reltés par un planteur de Bras-Panon en mars 2002, transmis par la FDGDON au SPV (E. Jeuffrault), puis reçus par S. Quilici du CIRAD (M. Lacroix det.). Depuis 2002, les récoltes faites aux piégeages lumineux tenus par le SPV à l'aéroport et au port de novembre à janvier sont régulières. Elles sont indiquées au tableau I. 
Fig. 7. - Carte des sites de récolte d'Alissonotum piceum besucheti à l'île de la Réunion (2001-2006)

(étoiles gris clair : imagos; étoile gris sombre : larves et imagos).

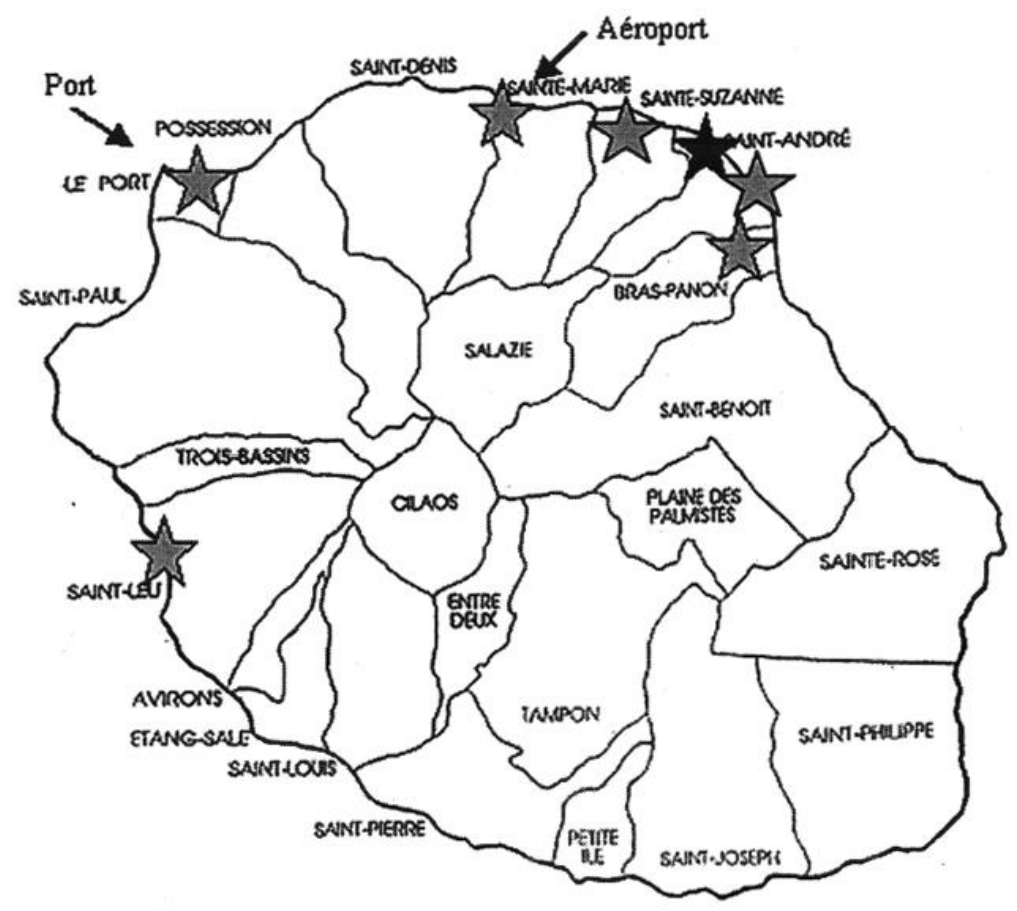

Tableau I. - Nombre d'adultes d'A. piceum besucheti récoltés aux pièges lumineux installés à l'aéroport et au port (Réunion, $2002-2006$ ).

\begin{tabular}{|l|c|c|c|c|c|}
\hline $\begin{array}{c}\text { Campagne/mois } \\
\text { Lieu }\end{array}$ & nov 2002-jan 03 & nov 2003-jan 04 & nov 2004-jan 05 & nov 2005-jan 06 & nov 2006 \\
\hline Aéroport & 7 & 34 & 3 & 18 & 19 \\
\hline Port & - & 4 & 5 & 2 & - \\
\hline
\end{tabular}

D'autres adultes ont été observés aux lumières des habitations sur la côte nord-est (Sainte-Marie à Saint-André), ainsi que dans une cocoteraie de Sainte-Suzanne.

Le tableau II mentionne la taille des adultes collectés, mesurée à l'aide d'une binoculaire munie d'un réticule $(\mathrm{G} \times 6,3)$.

Tableau II. - Mensurations (en mm) de plusieurs échantillons d'adultes d'A. piceum besucheti, récoltés au piège lumineux installé à l'aéroport de Gillot (Réunion).

\begin{tabular}{|c|c|c|c|c|c|c|c|}
\hline \multirow{2}{*}{$\begin{array}{c}\text { Date de } \\
\text { dissection }\end{array}$} & \multirow{2}{*}{ Référence } & \multicolumn{3}{|c|}{ Mâles } & \multicolumn{3}{c|}{ Femelles } \\
\cline { 3 - 8 } & & nb & L (extrêmes) & l & nb & L (extrêmes) & 1 \\
\hline 26.1.2005 & Gillot vol 2002/03 & 3 & $11,4(10,8-12,3)$ & 5,9 & 4 & $12,5(12,1-13,6)$ & 6,8 \\
\hline 24.I.I.2005 & Gillot vol 2003/04 & 0 & - & - & 26 & $12,6(\mathrm{nd})$ & 6,8 \\
\hline 16.III.2006 & Gillot vol 2005/06 & 8 & $12,3(12,1-13,6)$ & 6,5 & 10 & $13,2(12,5-15,3)$ & 7,2 \\
\hline
\end{tabular}

$1,2,3$ Les mesures ont été effectuées après une conservation en congélation de ${ }^{1} 2$ ans, de ${ }^{2} 1$ an ou de ${ }^{3} 4$ mois. Pour comparaison, DECHAMBRE (1986): đ̋, 12-13 mm; ㅇ, 12-14 mm; ENDRÖDI (1985): sexes confondus, 12-14 mm.

\section{Eléments de biologie}

Quelques espèces de Dynastidae sont connues en zone tropicale comme ravageurs des cultures (certains Oryctes, Heteronychus, etc.), mais depuis peu la connaissance s'est affinée et des espèces utiles ont aussi été observées (par ex., le genre Pentodon à Madagascar, Ratnadass et Randriamanantsoa, comm. pers., 2005) jouant un rôle identique aux vers de terre dans le recyclage de la matière organique ou la structuration du sol (espèces dites "ingénieurs du sol"). Il convient donc d'analyser les connaissances antérieures obtenues sur le genre ou l'espèce nouvellement introduite et de les confronter à nos observations pour mieux évaluer le statut de ce ver blanc du point de vue agronomique dans le cadre de l'entomofaune locale. 


\section{Etudes menées sur Alissonotum spp. dans le monde}

D'un point de vue systématique, si la tribu des Pentodontini est très riche en espèces (40\% des Dynastides, d'après ENDRÖDI, 1985), le genre Alissonotum Arrow, 1908 ne comprend qu'une espèce dans la région paléarctique, 8 dans la région orientale et 2 espèces récemment décrites (DUPUIS, 1998) dans la région africaine (Gabon). Des études menées en Asie et en Extrême-Orient sont régulièrement consacrées aux Alissonotum (PEREZ et al., 1980; HSIEH, 1981 ; WANG \& LI, 1987; LIU \& WANG, 1989; TSAI \& CHI, 1991 ; CHENG, 1991 ; LIU et al., 1996; SHARMA, 1985 et 1999 ; ABDULlaH et al., 2006). Les espèces les plus citées sont $A$. impressicole (longueur $\mathrm{L}=14-16 \mathrm{~mm}$ ) sur Canne à sucre (Inde, Vietnam, Taïwan, Chine) et $A$. simile (L $=10-11 \mathrm{~mm}$ ) sur Maïs et Riz pluvial (Inde).

Les Dynastidae ont une biologie qui diffère de celle des Melolonthidae par de nombreux points. S'il n'y a chez la plupart des espèces tropicales qu'une génération par an, c'est le stade adulte des Dynastidae qui a la plus grande longévité ( $c f$. infra). Les durées des stades larvaires sont beaucoup plus courtes que chez les Melolonthidae. Cela se traduit par une stratégie différente pour la reproduction de l'espèce. Les larves des Dynastidae n'ont qu'un faible niveau de réserves lipidiques (fig. 5) et les adultes doivent s'alimenter avant de pouvoir se reproduire, ce qui n'est pas le cas chez les Melolonthidae. L'apparition des adultes en début de saison humide leur permet de trouver des cultures au stade plantule ou jeune talle (sur Canne, des boutures en train de germer). Après cette période d'alimentation, qui peut être assez longue, l'accouplement pourra avoir lieu, la maturation des œufs étant achevée. Le nombre d'œufs pondus par femelle est variable, car tributaire de la qualité de la phase alimentaire. En général, ce nombre varie entre 30 et 60 .

Les principales données sur la dynamique des populations d'A. piceum besucheti proviennent de Maurice.

\section{Etudes menées sur Alissonotum piceum besucheti à Maurice}

Dans cette île, ce bio-concurrent a d'abord été mentionné sous le nom d'A. piceum (Box, 1953). Cette appellation a concerné également un autre Dynastide, Heteronychus licas, jusqu'en 1980 (MSIRI, 1981). La première constatation de dégâts dus à ces insectes date de 1958 (WIEHE, 1959).

Toutes les plantes-hôtes d'A. piceum besucheti ne sont pas connues, bien que, selon la littérature, elles appartiennent aux Poaceae (= Graminées). A Maurice, ce Dynastide est maintenant régulièrement cité sur Canne à sucre (MSIRI, 1991, 2001 à 2004), où il provoque des dégâts localisés sur quelques hectares (boutures trouées, bourgeons et base des pousses détruites par les adultes, racines coupées par les larves). Des deux stades, c'est l'adulte le plus nuisible et qui a la durée de vie la plus longue (on le capture presque toute l'année au piège lumineux, sauf de juin à août).

Lors de l'enquête menée de décembre 1989 à février 1991 par le MSIRI, 7150 trous ont été creusés dans les champs de canne (10 trous/parcelle, dimensions : $50 \times 100 \mathrm{~cm}^{2}$, centrés sur la souche et perpendiculaires à la ligne des pieds de canne). Un total de 9618 larves ont été collectées, dont 3,6\% correspondait à $A$. piceum. La densité moyenne était de 0,05 individu par trou; aucune larve n'a été trouvée dans les sols rocheux et ferralitiques humifères, les densités les plus fortes (10 à 15\% des populations) se situant dans les sols complexes de pentes de montagnes, les argiles noires magnésiennes et les sols ferralitiques faiblement humifères. Il n'y a qu'une génération par an (RAJABALEE, 1991). A. piceum besucheti est plus abondant en zone sub-humide ( $<1500 \mathrm{~mm}$ de pluies).

Lors de l'enquête du MSIRI, les récoltes d'adultes, effectuées au piège lumineux, étaient variables, mais très faibles, avec 3 maxima allant croissant, sans que cela soit lié à des générations successives : septembre ( 1 individu en moyenne/jour), puis février-mars (trois fois 
plus) et mai (sept fois plus). Lors des échanges dus au protocole technique franco-mauricien ( $c f$. infra), ces prises sont plus importantes mais sont restées réduites sur les 5 sites mis en œuvre durant les campagnes 2004/05 et 2005/06). Les prises ont totalisé quelques unités en novembre (sur 1 à 3 sites) et quelques dizaines d'individus en décembre et janvier ( 2 à 5 sites). L'heure des premiers vols est en partie liée à l'heure du coucher du soleil, comme dans le cas d'Hoplochelus marginalis ${ }^{2}$. En janvier, la plage de vol d'A. piceum $(19 \mathrm{~h} 30$ 19 h 40), à Maurice, est comprise dans celle de H. marginalis (19 h 20-19 h 50) à la Réunion (RAJABALEE, 1991 ; VerCAMBRE, 1986).

\section{Premières observations d'Alissonotum piceum besucheti à la Réunion}

Les larves âgées (L3) d'A. piceum besucheti sont observées, à Maurice, de juin à novembre (RAJABALEE, 1991). Si l'insecte parvenait à se multiplier à la Réunion, il y aurait un risque de confusion des larves lors des enquêtes menées sur Hoplochelus marginalis de juin à août (Melolonthidae).

Des prospections réalisées en partenariat avec la FDGDON en juillet 2005 ont permis de découvrir sur le terrain des larves des deux espèces et de repérer ainsi la première parcelle de canne à sucre infestée par cet Insecte. Elle est située dans la zone de Bois-Rouge (SaintAndré, altitude $10 \mathrm{~m}$ ), dans un champ qui a été replanté en avril 2005 avec la variété $R 579$. Le sol est humide et argileux. Alors que le 27 octobre 2005, la population était entièrement sous forme larvaire aux deux derniers stades (L2 + L3), on n'en relevait que $43,8 \%$ un mois plus tard, tandis que les nymphes et les adultes représentaient respectivement $4,1 \%$ et $52,1 \%$ des effectifs. Fin mars 2006 , la population est composée de $100 \%$ d'adultes.

Des mesures de taille et des dissections ont été également faites sur les adultes récoltés, dont les résultats apparaissent au tableau III.

Tableau III. - Mensurations (mm) et poids (g) d'échantillons frais d'adultes d'A. p. besucheti (Saint-André, 2006).

\begin{tabular}{|c|c|c|c|c|c|c|c|c|c|c|}
\hline \multirow{2}{*}{$\begin{array}{l}\text { Date de } \\
\text { dissection }\end{array}$} & \multirow[t]{2}{*}{ Référence } & \multicolumn{4}{|c|}{ Mâles } & \multicolumn{4}{|c|}{ Femelles } & \multirow[b]{2}{*}{ Observations } \\
\hline & & $\mathrm{nb}$ & L (extrêmes) & 1 & poids & $\mathrm{nb}$ & $\mathrm{L}$ (extrêmes) & 1 & poids & \\
\hline 5.IV.2006 & $\begin{array}{c}\text { Bois-Rouge } \\
\text { nov. } 2005\end{array}$ & 4 & $12,8(12,1-$ & 6,8 & 0,17 & 5 & $15,1)$ & 7,1 &, 18 & $\begin{array}{l}\text { Dissection d'adultes issus de } \\
\text { larves mises en élevage }\end{array}$ \\
\hline 1.IV.2006 & $\begin{array}{c}\text { Bois-Rouge } \\
\text { mars } 2006\end{array}$ & 5 & $12,2(11,5-12,6)$ & 6,5 & 0,19 & 9 & $14,2(13,4-15,3)$ & 7,2 & 0,28 & $\begin{array}{l}\text { Dissection juste après prélè- } \\
\text { vement des adultes au champ }\end{array}$ \\
\hline
\end{tabular}

Compte tenu des faibles effectifs, les données sur la taille sont cohérentes avec celles des échantillons capturés au piège lumineux et conservés au congélateur. La différence de poids entre les femelles appartenant aux deux échantillons de Bois-Rouge est probablement due à ce que les individus pris au champ en mars se sont normalement alimentés. C'est confirmé par l'état des ovaires: aucun développement pour les femelles issues des larves L3 prélevées en novembre 2005, alors que les ovocytes commencent à apparaitre (légères constrictions dans les ovarioles) chez les femelles prises directement au champ en mars 2006. Enfin, aucune de ces femelles ne s'était encore accouplée (bourse copulatrice vide).

La densité était relativement élevée dans la zone de prélèvement: 10 à 20 individus $/ \mathrm{m}^{2}$. Malgré cette charge importante, la culture en début à mi-montaison, stade végétatif particulièrement sensible de la canne, ne semblait pas souffrir dans sa croissance, confirmant ainsi que la plupart des larves terricoles des Dynastidae ne se nourrissent pas toujours de racines. Les jeunes larves se situaient en surface, alors que les stades plus âgés se trouvaient entre 20 et $25 \mathrm{~cm}$ de profondeur. Le traitement réalisé au Suxon ${ }^{\otimes}$ (à base de Chlorpyriphos-éthyl, $30 \mathrm{~kg}$ PC /ha), dirigé contre $H$. marginalis, ne semble pas avoir eu d'impact sur la population d' $\boldsymbol{A}$.

\footnotetext{
${ }^{2}$ Autre "ver blanc" introduit à la Réunion très probablement en 1973, ayant provoqué de gros dégâts et faisant encore l'objet d'un arrêté de lutte obligatoire, à base d'un biocide composé de spores de Beavveria sp., champignon entomopathogène.
} 
piceum besucheti, ce qui confirme que l'Insecte ne vit pas directement au-dessous ou sur les côtés de la souche où se trouve localisé l'insecticide à la plantation. De même, une étude réalisée en conditions de laboratoire a montré que le Beauveria sp., utilisé dans la lutte contre $H$. marginalis, n'était pas virulent vis-à-vis des adultes de ce nouveau ver blanc, confirmant ainsi sa spécificité.

\section{Hypothèses sur les voies d'introduction}

La mondialisation des échanges a pour effet une dissémination accrue d'organismes divers, parmi lesquels des Insectes. Dans le cas présent, les conséquences économiques semblent heureusement limitées. Mais il paraît utile de résumer les données recueillies afin de déterminer les voies possibles de cette colonisation récente en vue d'en éviter d'autres, plus graves.

Compte tenu des spécificités morphologiques de la sous-espèce, sa provenance ne peut être que l'île Maurice, seul pays où elle est signalée. D'autre part, cette introduction semble être récente (peu avant 2000 ?), compte tenu de la date de la première observation au piège lumineux, de la localisation (fig. 7) et du faible niveau des populations actuelles.

Sa présence près du port (la ville de Le Port) et de l'aéroport (Saint-Denis / Sainte-Marie) laisse penser que les échanges aéroportuaires sont probablement en cause. Mais ceux-ci sont très anciens entre les deux îles et la détection des imagos est facilitée par la mise en place de dispositifs lumineux particuliers.

En effet, depuis 1991, un protocole technique concernant les mesures à prendre pour empêcher la dissémination des Hannetons et autres Scarabéides a été signé entre la Réunion et la République de Maurice. L'objectif est d'exercer une surveillance particulière pendant la période de vol des adultes (du $1^{\text {er }}$ novembre au 31 janvier) afin d'éviter le transport des imagos lors des échanges touristiques et commerciaux entre les deux îles. Une note annuelle, ainsi que des avis aux usagers, sont aussi élaborés et diffusés auprès des opérateurs économiques et des administrations pour faire connaître ces mesures (DAF/SPV, 1997). Ces dispositions ont été prises après que l'on ait constaté :

a) la présence d'adultes de Scarabaeoidea (Hoplochelus marginalis dans le sens RéunionMaurice et Heteronychus licas dans le sens Maurice-Réunion) sur les ponts des bateaux accostant dans ces deux îles, lorsqu'ils ont séjournés dans les ports respectifs durant le vol annuel (VERCAMBRE, 1989);

b) la présence d'Hoplochelus marginalis, plus ou moins discutée, dans des soutes d'avions.

Le fait que la première observation d'A. piceum besucheti ait été faite à l'aéroport renforcerait l'hypothèse de son arrivée via les transports aériens. Il a également été constaté que la période de vol d'A. piceum besucheti est plus longue que celle d' $H$. marginalis, ce qui a pour effet de laisser une période sans surveillance (février à mai). Mais sa présence à Saint-Leu deux ans plus tard fragilise la simplicité de cette seule hypothèse. De plus, une expérimentation réalisée en plaçant des adultes d' $H$. marginalis sur le pont d'un bateau dans des conditions proches de la réalité, a montré que les adultes restaient prostrés et qu'aucun envol n'était enregistré le soir et la nuit ; les va-et-vient des dockers et la température élevée du lendemain entraînaient une mortalité rapide des Coléoptères par écrasement ou par voie naturelle (VERCAMBRE, 1993).

Il est donc malaisé de déterminer avec certitude les voics et les causes de la présence d'A. piceum besucheti à la Réunion, d'autant plus que cette espèce n'était pas la plus attendue, compte tenu de sa situation dans le pays d'origine. Même si le transport par voie aérienne semble le plus probable, il est toujours étonnant de constater qu'une introduction puisse se faire par ce seul vecteur, alors que des relations aériennes régulières existent depuis plus de 40 ans. Pour le transport maritime, c'est encore plus ancien et la faune malgache, qui est très riche en ce type d'Insectes, n'a pas fait tache d'huile sur les îles voisines. 
Il est très probable que ce Dynastide ait été introduit avec de la terre contenue dans des bacs ou des pots de fleurs où vivaient des larves et/ou des nymphes, ainsi que cela a dû être le cas pour $H$. marginalis en juin-août 1973.

Une analyse des échanges entre Maurice et la Réunion où la présence de terre, habituellement interdite, pourrait être invoquée (pépiniéristes, Floralies...), une connaissance plus fine de sa répartition à Maurice et du comportement des adultes (plantes-hôtes, résistance à la dessiccation, niches de repos durant la journée par exemple) pourraient nous donner une des clés du mystère.

\section{Conclusion}

D'après l'ensemble des données consultées, il semble que cette nouvelle espèce puisse être capable de dégâts significatifs sur Canne à sucre, mais fort heureusement assez localisés, si l'on considère ses preferenda pédologiques (RAJABALEE et al., 1995).

Il reste à le vérifier dans différents sites de la Réunion propices à son développement, et à étudier la sensibilité d'autres cultures moins vigoureuses (Maïs, Riz pluvial...).

Destinée aux services agricoles de développement et aux planteurs, une fiche illustrée d'identification des larves et des adultes sera établie, pour éviter toute confusion entre A. piceum besucheti et $H$. marginalis.

Plus généralement, cette nouvelle introduction, dont les voies sont difficiles à préciser avec certitude, pose le problème récurrent des échanges internationaux, dont l'intensification, en quantité, en rapidité et en diversité, permet la dissémination d'organismes qui étaient localisés et peu communs.

REMERCIEMENTS. - Les auteurs adressent leurs vifs remerciements à B. Reynaud (CIRAD), E. Jeuffrault (SPV), D. Fontaine (CIRAD) et A. Robert (FDGDON) pour avoir rendu possible ces observations.

\section{AUTEURS CITÉS}

ABDUllah M., BisWAS M.M. \& SidDiQUeE M.N.A., 2006. - Responses of some promising clones to major insect pests of sugarcane. Indian Journal, 56 (3): 23-28.

Box H.E., 1953. - List of sugar-cane insects. Ed. Commonwealth Institute of Entomology, London, $101 \mathrm{p}$.

CHENG W. Y., 1991. - Research and development of sugarcane insects in Taiwan. Chinese Journal of Entomology, special Publication, 7. Proceedings of A Symposium on the Advances in the Entomology of Taiwan: 167-181.

DAF/SPV, 1997. - Application du protocole d'accord franco-mauricien de surveillance des introductions de hannetons/scarabées - période du 01/11/96 au 31/01/97, 8 p. et annexes.

DeCHAMBRE R.-P., 1986. - Insectes Coléoptères Dynastidae. Faune de Madagascar, 65. Paris : Muséum national d'Histoire naturelle, $215 \mathrm{p}$.

DupuIs F., 1998. - Les espèces africaines du genre Alissonotum Arrow, 1908. Revue Française d'Entomologie, 20 (3) : 115-119.

ENDRÖDI S., 1977. - Alissonotum piceum besucheti subsp n. (Dynastidae). www.insects.demo.co.uk/ coleo/dynastinae.html

1985. - The Dynastinae of the world. Séries Entomologica, vol. 28. K.A. Spencer Ed., Dr W. Junk Pub., 800 p., 46 planches.

HSIEH S.A., 1981. - Seasonal abundance and movement of sugarcane white grub, Alissonotum impressicole Arrow in relation to soil moisture. Report of the Taiwan Sugar Research Institute, 93: 39-49.

LIU X.L. \& WANG J.X., 1989. - Preliminary studies on pathogenicity on different strains of the nematode Steirnema glaseri Steiner to the sugarcane beetle Alissonotum impressicole Arrow. Natural Enemies of Insects, 3 (11):101-103, 109.

LIU Z.C.., LIU J. F., WANG C. X. \& YANG W. H., 1996. - The role of biological control in integrated control of sugarcane insect pests. International Symposium on the Use of Biological Control Agents under Integrated Pest Management, Taipei, Taiwan. 
MSIRI, 1981-2004. - Annual report (Mauritian Sugar Industry Research Institute), report 1981: 47; 1991, Annual report 1990:51;-2001, Annual report $2000: 30 ;-2002$, report $2001: 29 ;-2003$, report 2002: 32;-2004, report $2003: 35$.

PeReZ J.R., VAN K. N., CASTRO S., KHU N.V. \& VAN T.T., 1980. - Disease and pests of sugarcane in the socialist republic of Vietnam. Ciencias de la Agricultura, $6: 156-158$.

RAJABALEE A., 1991. - Etudes sur les vers blancs (Coleoptera : Scarabaeidae) de la canne à l'île Maurice. Rencontres Caraïbes en lutte biologique, Guadeloupe 5-7 novembre 1990. Paris: ed. INRA (les Colloques, $\mathrm{n}^{\circ} 58$ ) : 69-76.

Rajabalee A., Govendasamy M. \& Ganeshan S., 1995. - Proceedings XXI Congress of the International Society of Sugar Cane Technologists, Bangkok, Thailand, 5-14 March, 1992 : 609-620.

ROUILLARD G., 1990. - Historique de la canne à sucre à l'île Maurice (1639-1989). $50 \mathrm{p}$.

SHARMA S., 1985. - Scarabaeid beetle outbreak in upland rice. International Rice Research Newsletter, 10 (1): 24 .

— 1999. - Black beetle Alissonotum simile (Coleoptera : Dynastinae) : a new pest on maize in Nagaland. Insect Environment, 4 (4): 131-132.

TSAI S.M. \& CHI H., 1991. - Two-sex life tables of the sugarcane black beetle, Alissonotum impressicolle Arrow (Coleoptera: Scarabaeidae). Report of the Taiwan Sugar Research Institute, 134 : 1-18.

VERCAMBRE B., 1986. - Rapport IRAT-Réunion, document polycopié: p. 41.

_ 1989. - Note au sujet des insectes, notamment de scarabées, capturés sur les bateaux entrant à l'île de la Réunion (15/12 au 18/12/1989), document polycopié : 10 p., 7 annexes.

— 1993. - Etude du comportement du stade adulte de Hoplochelus marginalis (Coleopt. Melolonthinae) embarqué sur un bateau. Note d'étude, document polycopié, 4 p., 3 tabl., 4 fig., 1 annexe, 3 planches photo.

WANG J.X. \& Li L.Y., 1987. - Entomogenous nematodes research in China. Revue de Nématologie, $10(4): 483-489$.

WIEHE P.O., 1959. - Résumé du rapport annuel de l'Institut de Recherches Sucrières pour 1958. Ed. The General Printing \& Stationary Company $L^{\mathrm{d}}$, Port Louis, $98 \mathrm{p}$.

WILLIAMS J.R. \& MAMET J.R., 1962. - The insects and other invertebrates of sugar cane in Mauritius and Reunion. Mauritian Sugar Industry Research Institute, Occasional Paper, 8, 23 p. (www.nhm.org/ research/entomology/coleoptera/scarabaeidae/dynastinae.htlm)

\section{MÉMOIRES DE LA SEF}

Vient de paraûtre, août 2007
Les MÉMOIRES DE LA SEF, nº 7, 2007,

"TETRACHA (Coleoptera, Cicindelidae, Megacephalina). Révision du genre et descriptions de nouveaux taxons" par Roger NAVIAUX,

198 p., 83 pl. de figures, 14 pl. coul.

Prix : 60 euros (45 euros aux membres de la SEF).

Rappel des précédents numéro : voir Bull. Sc. Ent. Fr., 112 (1): 2. 